\title{
17AAG-induced internalisation of HER2-specific Affibody molecules
}

\author{
LOVISA GÖSTRING ${ }^{1}$, STURE LINDEGREN $^{2}$ and LARS GEDDA ${ }^{1,3}$ \\ ${ }^{1}$ Department of Immunology, Genetics and Pathology, Uppsala University, SE-75185 Uppsala; \\ ${ }^{2}$ Department of Radiation Physics, Sahlgrenska Academy, University of Gothenburg, SE-41345 Gothenburg; \\ ${ }^{3}$ Swedish Radiation Safety Authority, SE-17116, Stockholm, Sweden
}

Received August 24, 2015; Accepted June 16, 2016

DOI: $10.3892 / 01.2016 .4990$

\begin{abstract}
The geldanamycin derivative 17-allylamino17-demethoxygeldanamycin (17-AAG) is known to induce internalisation and degradation of the otherwise internalisation-resistant human epidermal growth factor receptor 2 (HER2) receptor. In the present study, 17-AAG was used to increase internalisation of the HER2-specific Affibody molecule ABY-025. The cellular redistribution of halogen-labelled ${ }^{211}$ At-ABY-025 and radiometal-labelled ${ }^{111}$ In-ABY-025 following treatment with $17-\mathrm{AAG}$ was studied. 17-AAG treatment of SKOV-3 human ovarian carcinoma and SKBR-3 human breast carcinoma cells to some extent shifted the localisation of ${ }^{111}$ In-ABY-025 from the cell surface to intracellular compartments in the two cell lines. ABY-025 labelled with the high-linear energy transfer $\alpha$ emitter ${ }^{211}$ At was also internalised to a higher degree; however, due to its physiological properties, this nuclide was excreted faster. The results indicate that 17-AAG may be used to facilitate cell-specific intracellular localisation of a suitable cytotoxic or radioactive agent coupled to ABY-025 in HER2-overexpressing cells.
\end{abstract}

\section{Introduction}

The epidermal growth factor receptor (EGFR) family of growth receptors, consisting of EGFR, human epidermal growth factor receptor (HER)2, HER3 and HER4 (also known as ErbB1-4, respectively), is known to be involved in a number of cancer types. Therefore, these receptors, particularly EGFR and HER2, are important targets for tumour diagnostics and therapy. HER2-overexpression is most common in breast and ovarian cancers, and is also associated with poor prognosis (1).

Correspondence to: Professor Lars Gedda, Department of Immunology, Genetics and Pathology, Uppsala University, Dag Hammarskjölds v 20, SE-75185 Uppsala, Sweden

E-mail: lars.gedda@igp.uu.se

Key words: HER2, 17-AAG, Affibody molecule, radiometal, radiohalogen
Upon ligand binding, EGFR forms a dimer, which is internalised via endosomes and eventually degraded in lysosomes $(2,3)$. However, HER2, which has no natural ligand and therefore is normally dependent on heterodimerisation with other members of the HER family of receptors, is not internalised and degraded in this way. Certain authors have reported that this receptor continuously circulates between the cell membrane and early endosomes (4), whereas others regard HER2 as an internalisation-resistant receptor that is actively excluded from clathrin-coated pits (5). This may be part of the reason why HER2 signalling is so potent $(6,7)$.

However, HER 2 may be forced into internalisation and degradation using the ansamycin antibiotic geldanamycin (GA) or its derivative 17-allylamino-17-demethoxygeldanamycin (17-AAG) (8). By blocking its ATP-binding site, these compounds inhibit heat shock protein 90 (Hsp90), a chaperone required for stabilisation of HER2 at the cell membrane (9). Without an active Hsp90 chaperone, HER2 is ubiquitinylated by the E3 ubiquitin ligase CHIP $(10,11)$ and internalised in a proteasome-dependent process that ends in lysosomal degradation (12). It is not clear whether the increased internalisation results from the redirection of endosomes from recirculation to degradation in lysosomes (13), or from faster endocytosis followed by degradation (12).

Hsp90 acts as a chaperone for a number of other proteins involved in receptor signalling, including Raf of the Ras/mitogen-activated protein kinase pathway and p-Akt of the phosphoinositide-3 kinase (PI3K)/Akt pathway (14). Since these pathways are often upregulated in cancer cells, a feature believed to be associated with cytotoxic drug resistance $(15,16)$, impaired signalling may potentiate the effect of cytotoxic agents. 17-AAG, which is less toxic than geldanamycin, has shown agonistic tumour-cell killing effects in combination with, for instance, paclitaxel or carboplatin $(17,18)$ and $\gamma$ irradiation (19). 17-AAG (or formulated as KOS-953/Tanespimycin) has been recently evaluated in clinical trials (15).

The current study investigated the effects of 17-AAG on a radiolabelled HER2-binding Affibody molecule, ABY-025. Affibody molecules are three-helical proteins derived from the staphylococcal IgG-binding protein A. By randomising 13 amino acids in its binding site, it is possible to change the IgG-binding properties of the Affibody molecule and generate Affibody molecules specific for other proteins via phage 
display or other selection systems (20). Affibody molecules specific for EGFR and HER2 have shown promising results in tumour-targeting studies $(21,22)$.

The results indicate that 17-AAG treatment of SKOV-3 and SKBR-3 cells to some extent shifts the localisation of ${ }^{111}$ In-ABY-025 from the cell surface to intracellular compartments in both cell lines. ABY-025 labelled with the high-linear energy transfer (LET) $\alpha$ emitter ${ }^{211} \mathrm{At}$ is also internalised to a higher degree; due to its physiological properties, however, this nuclide is excreted faster and thus does not residualise intracellularly.

\section{Materials and methods}

Cell culture. SKOV-3 human ovarian carcinoma cells (ATCC, Manassas, VA, USA) were grown in McCoy's medium (Biochrom; Merck KGaA, Darmstadt, Germany) supplemented with $10 \%$ foetal bovine serum (Sigma-Aldrich, St. Louis, MO, USA), 2 mM L-glutamine (Biochrom; Merck KGaA), $100 \mathrm{U} / \mathrm{ml}$ penicillin and $100 \mu \mathrm{g} / \mathrm{ml}$ streptomycin (Biochrom; Merck KGaA). SKBR-3 cells (ATCC) were cultured in the same medium but with $20 \%$ foetal bovine serum.

Affibody molecules. The HER2-specific Affibody Cys- $Z_{2891}$ was used. This protein has a C-terminally located cysteine, which was utilised for maleimide coupling of Alexa Fluor ${ }^{\circledR} 488$ to the protein (as described below). When this cysteine is instead maleimide-coupled to DOTA, the Affibody is referred to as ABY-025 (23). Cys- $Z_{2891}$ and ABY-025 were kindly provided by Affibody AB (Solna, Sweden).

${ }^{211}$ At labelling of $A B Y-025$. The labelling was performed as previously described by Lindegren et al (24). In short, $250 \mu \mathrm{g}$ (35.7 nmol) of ABY-025 in 0.1 M borate buffer ( $\mathrm{pH} 8.5$ ) was mixed with $71.4 \mathrm{nmol}$ of m-MeATE in dimethyl sulfoxide (DMSO) and incubated for $30 \mathrm{~min}$ with gentle agitation. After elution with $0.2 \mathrm{M}$ sodium acetate buffer ( $\mathrm{pH}$ 5.5) on a NAP-5 column (GE Healthcare Life Sciences, Uppsala, Sweden), ABY-025-MeATE was added to $39.8 \mathrm{MBq}$ of ${ }^{211} \mathrm{At}$ (Rigshospitalet, Copenhagen, Denmark) which had been activated with N-iodosuccinimide (NIS) and incubated with agitation for $60 \mathrm{sec}$. More NIS was added, and the mixture was incubated for a further $60 \mathrm{sec}$. Sodium ascorbate was added in order to reduce unreacted astatine, and the ${ }^{211}$ At-labelled ABY-025 was purified on a NAP-5 column using phosphate-buffered saline (PBS) as eluent.

Specificity of ${ }^{211}$ At-ABY-025 uptake. Cells (25,000 of SKOV-3 and 100,000 of SKBR-3 per well) were seeded into 6-well plates and allowed to grow in complete medium for 5 days. Following $2 \mathrm{~h}$ of incubation with $2.3 \mathrm{nM}{ }^{211} \mathrm{At}-\mathrm{ABY}-025$ with or without $230 \mathrm{nM}$ unlabelled ABY-025, the cells were washed, trypsinised and measured in a gamma counter (Wizard 1480; Wallac Oy, Turku, Finland).

Uptake and internalisation of ${ }^{211}$ At-ABY-025 (acid wash assay). SKOV-3 and SKBR-3 cells were seeded into 6-well plates as described. The medium was replaced with $3 \mathrm{ml}$ of $2.3 \mathrm{nM}\left(=30 \times \mathrm{K}_{\mathrm{D}}\right)$ ABY-025 in complete medium with either 100 nM 17-AAG (A.G. Scientific, Inc., San Diego, CA, USA)

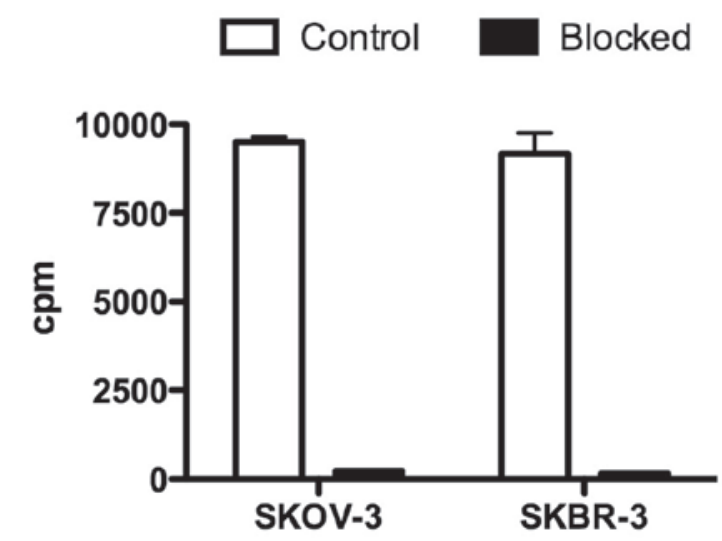

Figure 1. ${ }^{211} \mathrm{At}-\mathrm{ABY}-025$ specificity: Cellular uptake following incubation for $2 \mathrm{~h}$ with $2.3 \mathrm{nM}^{211} \mathrm{At}-\mathrm{ABY}-025$ ('control') or $2.3 \mathrm{nM}^{211} \mathrm{At}-\mathrm{ABY}-025+230 \mathrm{nM}$ unlabelled ABY-025 ('blocked'). cpm, counts per minute.

dissolved in DMSO, or the corresponding volume of DMSO (control). After 2, 4 and $6 \mathrm{~h}$, samples were taken using the acid wash internalisation assay (25): After two washes in serumfree medium, the cells were incubated with $0.5 \mathrm{ml}$ ice-cold acid (0.2 M glycine, $0.15 \mathrm{M} \mathrm{NaCl}, 4 \mathrm{M}$ urea, $\mathrm{pH}$ 2) on ice for $5 \mathrm{~min}$. The acid (with the cell surface fraction of ${ }^{211} \mathrm{At}$ ) was collected and cells were washed with additional $0.5 \mathrm{ml}$ acidic solution. The cells were treated with $1 \mathrm{M} \mathrm{NaOH}$ and removed from the petri dish using a cell scraper. This cell suspension was retained as the internalised fraction of ${ }^{211}$ At. For each time point, triplicates were used for every treatment. Radioactivity was measured in a gamma counter, with all samples in one reading.

${ }^{111}$ In labelling of $A B Y-025$. Labelling was performed as described previously (23). In short, $50 \mu \mathrm{g}$ of ABY-025 was diluted in $50 \mu 10.2 \mathrm{M}$ ammonium acetate buffer ( $\mathrm{pH} 5.3$ ), mixed with $50 \mathrm{MBq}{ }^{111} \mathrm{InCl}$ (Medtronic, Minneapolis, $\mathrm{MN}$, USA) and incubated at $60^{\circ} \mathrm{C}$ for $40 \mathrm{~min}$. Labelling yield was determined on chromatography strips (Biodex Medical Systems, Shirley, NY, USA) in 0.2 M citric acid and analysed in a Phosphor Imager (Cyclone Storage Phosphor System; PerkinElmer, Inc., Waltham, MA, USA).

Uptake and internalisation of ${ }^{111}$ In-ABY-025 (acid wash assay). Approximately 500,000 SKOV-3 or SKBR-3 cells were seeded into $3.5-\mathrm{cm}$ petri dishes and allowed to grow at least overnight. Cells were incubated with ${ }^{111}$ In-ABY-025 $\pm 17-A A G$, and surface-bound and internalised fractions were separated using acid wash as described. SKOV-3 cells were treated with 10 and 100 nM 17-AAG, while SKBR-3 cells were treated with $100 \mathrm{nM}$ only. Samples were taken at $0,1,3,5$ and $7 \mathrm{~h}$ after the start of incubation. Radioactivity was measured in a gamma counter, with all samples in one reading.

Alexa Fluor ${ }^{\circledR} 488$ labelling of Cys- $Z_{2891}$. Cys- $Z_{2891}(700 \mu \mathrm{g})$ was diluted to $100 \mathrm{nM}$ and reduced with $20 \mathrm{mM}$ dithiothreitol (DTT) for $45 \mathrm{~min}$ at $37^{\circ} \mathrm{C}$. DTT was removed in NAP-5 columns equilibrated in PBS, and $500 \mathrm{nmol}$ (5X molar excess) Alexa Fluor ${ }^{\circledR} 488$ C5-maleimide (Molecular Probes; Thermo Fisher Scientific, Inc., Waltham, MA, USA) dissolved in 
A

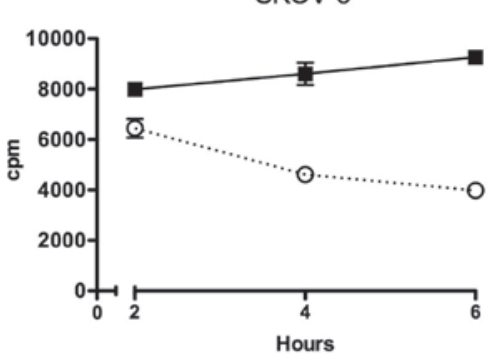

B

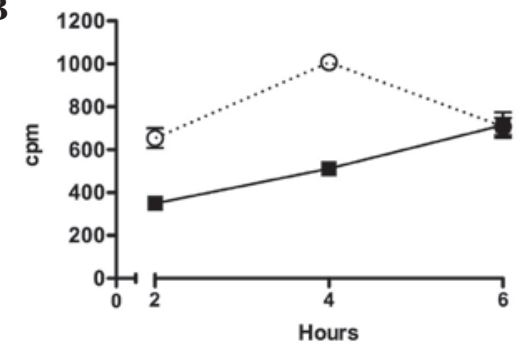

C

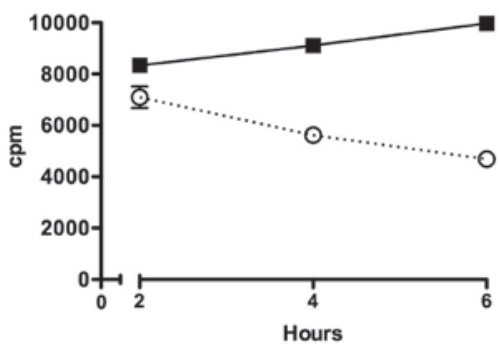

SKBR-3
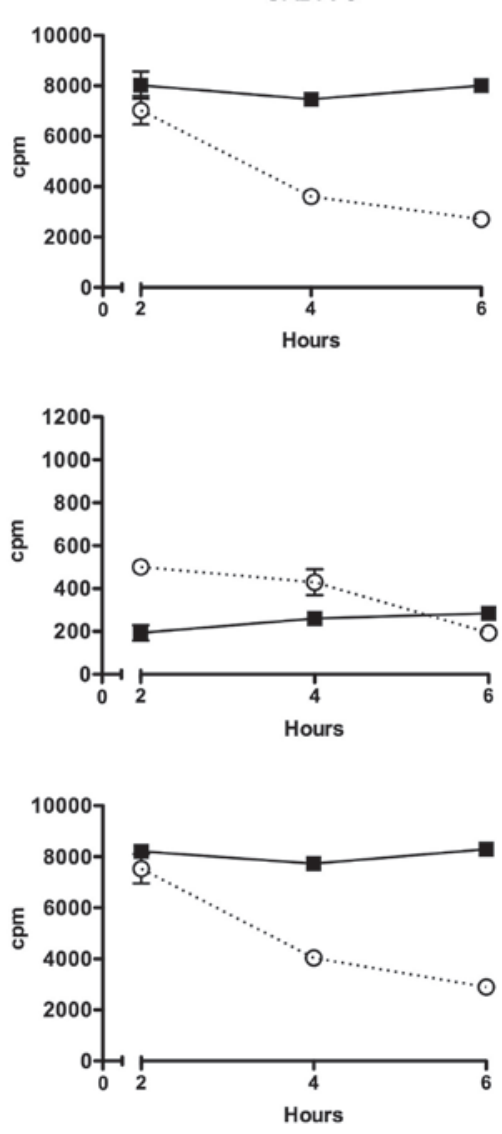

Figure 2. ${ }^{211}$ At-ABY-025 uptake in SKOV-3 (left column) and SKBR-3 (right column) cells. (A) Surface-bound and (B) intracellular ${ }^{211}$ At in untreated (full line) and $100 \mathrm{nM}$ 17-AAG-treated (dotted line) cells. (C) Total uptake (sum of the surface and intracellular fractions). Data are presented as the mean of three samples \pm standard deviation. $\mathrm{cpm}$, counts per minute.

DMSO was added. After incubation at $4^{\circ} \mathrm{C}$ overnight, unbound Alexa488 was removed in a PD-10 column (GE Healthcare Life Sciences) equilibrated with PBS. Degree of labelling and protein concentration were determined using a NanoDrop ND-1000 (Thermo Fisher Scientific, Inc., Wilmington, DE, USA).

Immunofluorescence microscopy. SKOV-3 (10,000 cells) and SKBR-3 (20,000 cells) were seeded into 8-chamber slides (Nunc 154534) and allowed to grow for 3 days. The medium was exchanged for complete medium with $200 \mathrm{nM}$ Alexa488-Z2891 and 100 nM 17-AAG, or the corresponding volume DMSO (control), and the cells were incubated for $3 \mathrm{~h}$ at $37^{\circ} \mathrm{C}$. The slides were gently washed twice with serum-free medium and the chambers removed. Following a quick wash in PBS, the cells were fixed in $4 \%$ formaldehyde for $15 \mathrm{~min}$ at room temperature. The slides were washed in PBS, incubated for $10 \mathrm{~min}$ in $2 \mu \mathrm{M}$ Hoechst 33342 and allowed to dry. They were then mounted in Vactashield Antifade Mounting Medium (Vector Laboratories, Inc., Burlingame, CA, USA). Images were taken with a Zeiss LSM 510 Meta confocal microscope.

Pre-incubation with ${ }^{111}$ In-ABY-025. SKOV-3 cells were incubated with ${ }^{111}$ In-ABY-025 as described above, and $100 \mathrm{nM}$ 17-AAG was added either immediately or after 0.5 or $2 \mathrm{~h}$. Samples were taken at different time points using the acid wash method described above.

\section{Results}

${ }^{211}$ At-ABY-025 specificity. ${ }^{211} \mathrm{At}-\mathrm{ABY}-025$ was shown to bind to SKOV-3 and SKBR-3 cells, and this binding was completely blocked with $230 \mathrm{nM}$ of ABY-025 (Fig. 1), confirming certain specificity.

Uptake and internalisation of ${ }^{211} A t-A B Y-025$. In untreated SKOV-3 cells, the amount of surface-bound ${ }^{211}$ At-ABY-025 increased slightly between 2 and $6 \mathrm{~h}$, whereas treatment with 17-AAG decreased cell surface location during the same time (Fig. 2). The intracellular concentration of ${ }^{211} \mathrm{At}$ was lower for treated and non-treated cells; however, the relative increase with time was higher in both cases. In SKBR-3 cells, the pattern was similar, but the surface uptake of untreated cells did not increase over time, and 17-AAG induced a faster reduction of surface-bound ${ }^{211}$ At-ABY-025 than in the SKOV-3 cells. Total cellular uptake was simply calculated as the sum of counts of the two fractions. Due to the relatively small intracellular fraction, the total value was fairly similar to that of the surface fraction.

Uptake and internalisation of ${ }^{111} I n-A B Y-025$. In untreated SKOV-3 cells, surface bound ${ }^{111}$ In-ABY-025 increased with time and reached a steady level after 5-7 h (Fig. 3). With $100 \mathrm{nM}$ 17-AAG present, the level of surface-bound ${ }^{111}$ In-ABY-025 stabilised at a lower level after $\sim 3 \mathrm{~h}$, whereas $10 \mathrm{nM}$ of 17-AAG had no detectable effect. The decreased 

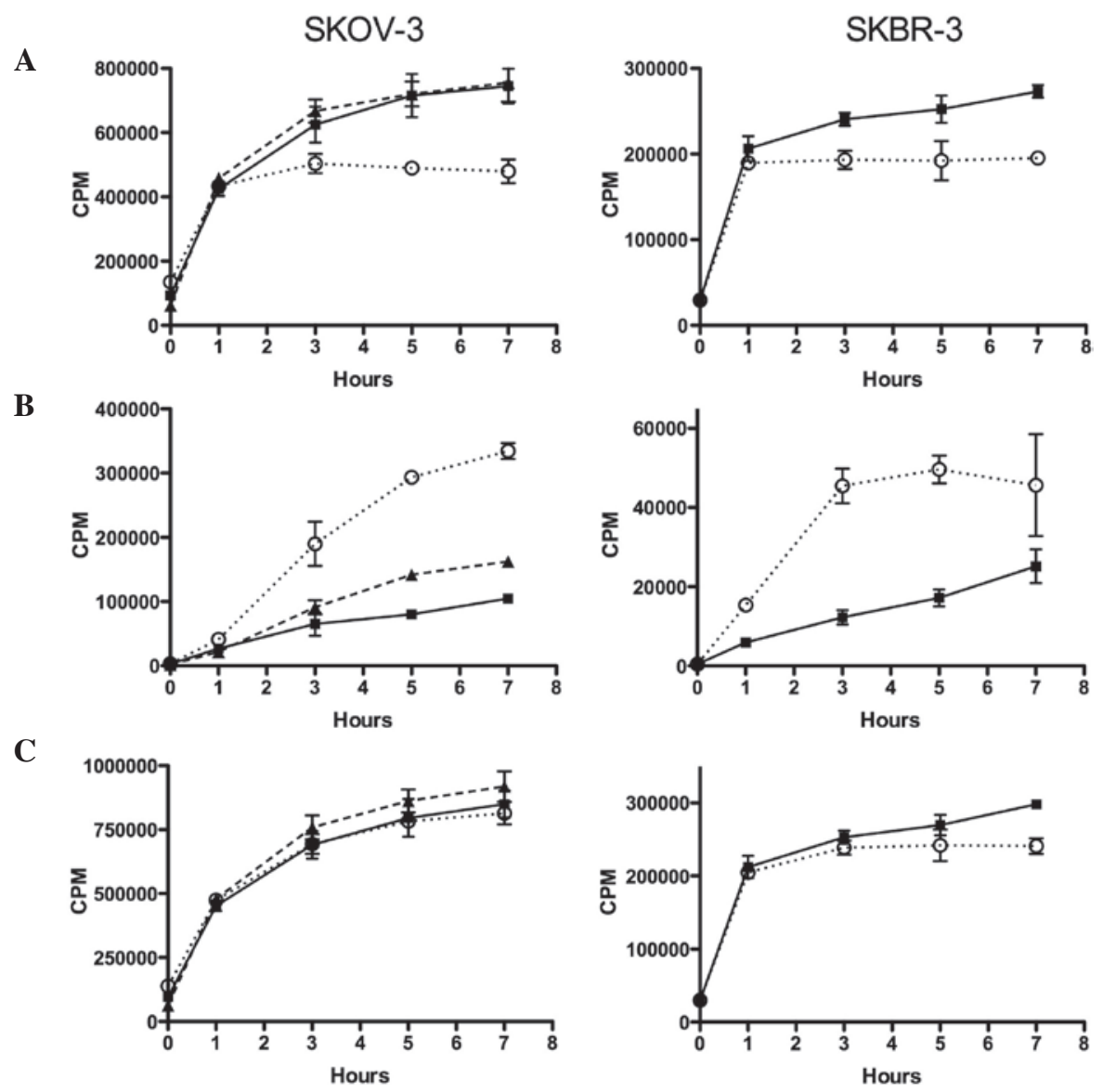

Figure 3. Uptake and internalisation of ${ }^{111}$ In-ABY-025 by SKOV-3 cells (left column) and SKBR-3 cells (right column) treated with 17-AAG [10 nM (dashed line; SKOV-3 only) or $100 \mathrm{nM}$ (dotted line with circles)] compared to control (full line). (A) Surface bound fraction; (B) intracellular fraction; (C) total cellular uptake of ${ }^{111}$ In (sum of the surface and intracellular fractions). Data is presented as the mean of three samples \pm standard deviation. cpm, counts per minute.

surface levels of ${ }^{111}$ In-ABY-025 observed with 100 nM 17-AAG corresponded to a continuous increase in intracellular ${ }^{111} \mathrm{In}$. A similar but weaker increase was observed with $10 \mathrm{nM}$ 17-AAG. In SKBR-3 cells, the pattern was similar. In cells treated with $100 \mathrm{nM}$ 17-AAG, the surface levels of ${ }^{111} \mathrm{In}-\mathrm{ABY}-025$ were stable from $\sim 1 \mathrm{~h}$, whereas this fraction continued to increase in untreated cells. The increased intracellular portion observed with $100 \mathrm{nM}$ 17-AAG was faster than in SKOV-3 cells. The total uptake was similar for 17-AAG-treated and untreated SKOV-3 and SKBR-3 cells.

Immunofluorescence staining with directly labelled Affibody molecules. Alexa488-labelled anti-HER2 Affibody molecules (Z2891) were incubated with SKOV-3 or SKBR-3 cells for $3 \mathrm{~h}$ in the presence or absence of $100 \mathrm{nM} 17-\mathrm{AAG}$. It was clearly observed that 17-AAG increased intracellular localisation; the fluorescence was could be detected mainly in vesicles around the nucleus (Fig. 4).

Pre-incubation with ${ }^{111} I n-A B Y-025$. Pre-incubation with ${ }^{111} \mathrm{In}-\mathrm{ABY}-025$ and the later addition of 17-AAG delayed internalisation somewhat; however, once 17-AAG was added, the internal uptake seemed to follow the same pattern as when 17-AAG was added simultaneously (Fig. 5). After 4-6 h, the intracellular fraction appeared to be similar in all 17-AAG-treated cells, independently of pre-incubation time $(0,0.5$ or $2 \mathrm{~h})$. The total amount of cell-bound ${ }^{111}$ In was approximately the same in all cases, as 17-AAG treatment led to reduced amounts of ${ }^{111} \mathrm{In}-\mathrm{ABY}-025$ at the cell membrane.

\section{Discussion}

The HER2 receptor is known to be resistant to internalisation followed by degradation, and is believed to also protect EGFR and HER 3 from internalisation when forming heterodimers with these receptors $(6,7)$. Hence, $17-\mathrm{AAG}$ may be used to reduce the number of receptors on the surface of HER2-overexpressing tumour cells. Furthermore, a number of downstream signalling proteins are dependent on the Hsp90 chaperone, and application of 17-AAG also leads to degradation of these proteins (14). A number of clinical studies on 17-AAG (or tanespimycin) have been published (26-28) (Www.clinicaltrials.gov).

As demonstrated in the present study, 17-AAG can also be used to increase the internalisation of a HER2-targeted molecule, such as the Affibody ABY-025. ${ }^{211}$ At-ABY-025 and ${ }^{111}$ In-ABY-025 were internalised to a higher extent with 17-AAG present, and were most likely directed to the lysosomes for degradation (12). However, the retention of the nuclides differed. As ${ }^{211}$ At is a halogen, it is rapidly excreted by the cells following lysosomal degradation of the Affibody. The radiometal ${ }^{111} \mathrm{In}$, on the other hand, is known to be trapped in the cell. This was observed when 17-AAG increased the amount of intracellular ${ }^{111}$ In without decreasing the total amount of cell-associated ${ }^{111}$ In (29). The difference in cellular retention 

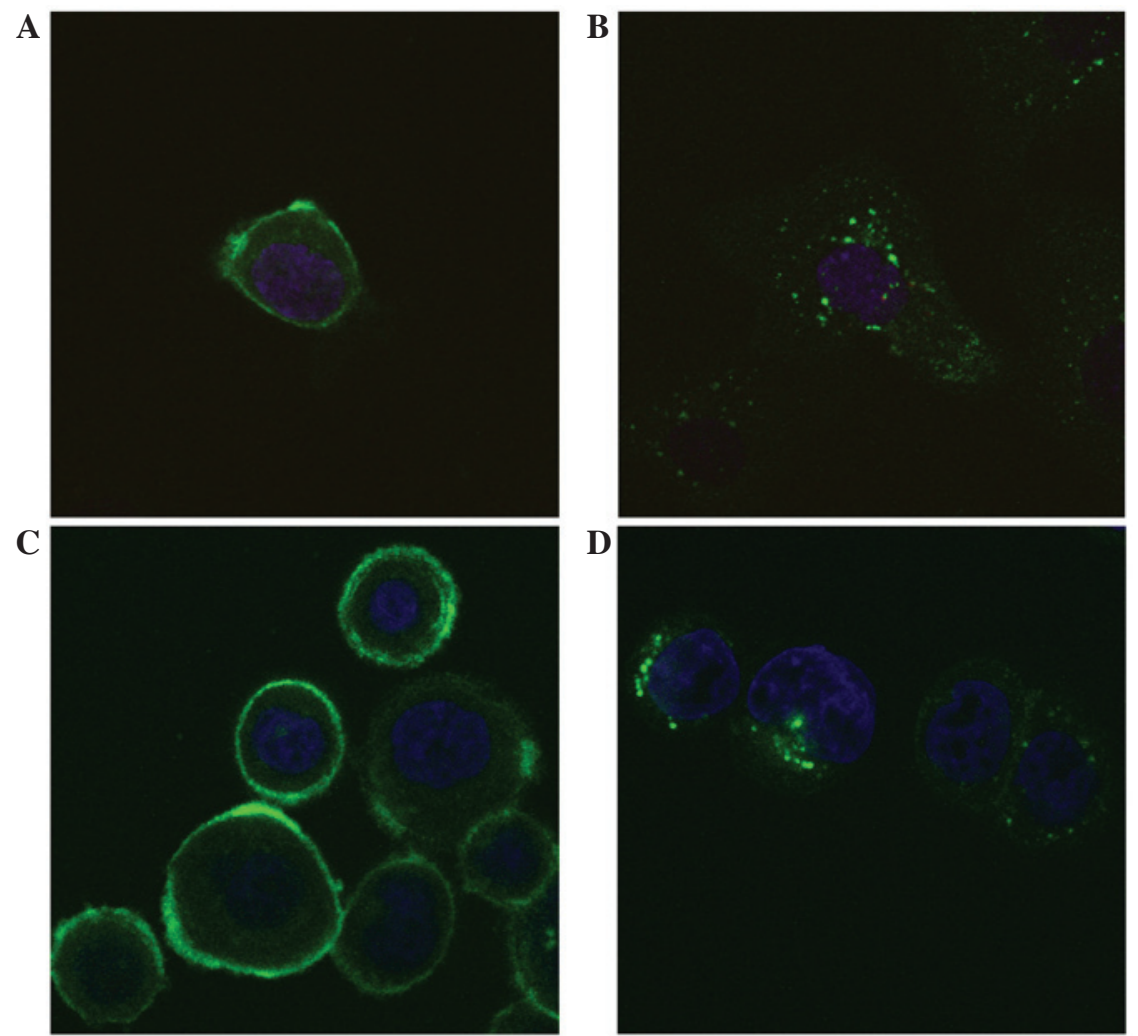

Figure 4. Immunofluorescence microscopy of (A and B) SKOV-3 and (C and D) SKBR-3 cells incubated in 200 nM Alexa Fluor ${ }^{\circledR}$ 488-labelled anti-HER2 Affibody molecules, (B and D) with or (A and C) without $100 \mathrm{nM}$ 17-AAG for 3 h. Nuclear staining was performed with Hoechst (blue).
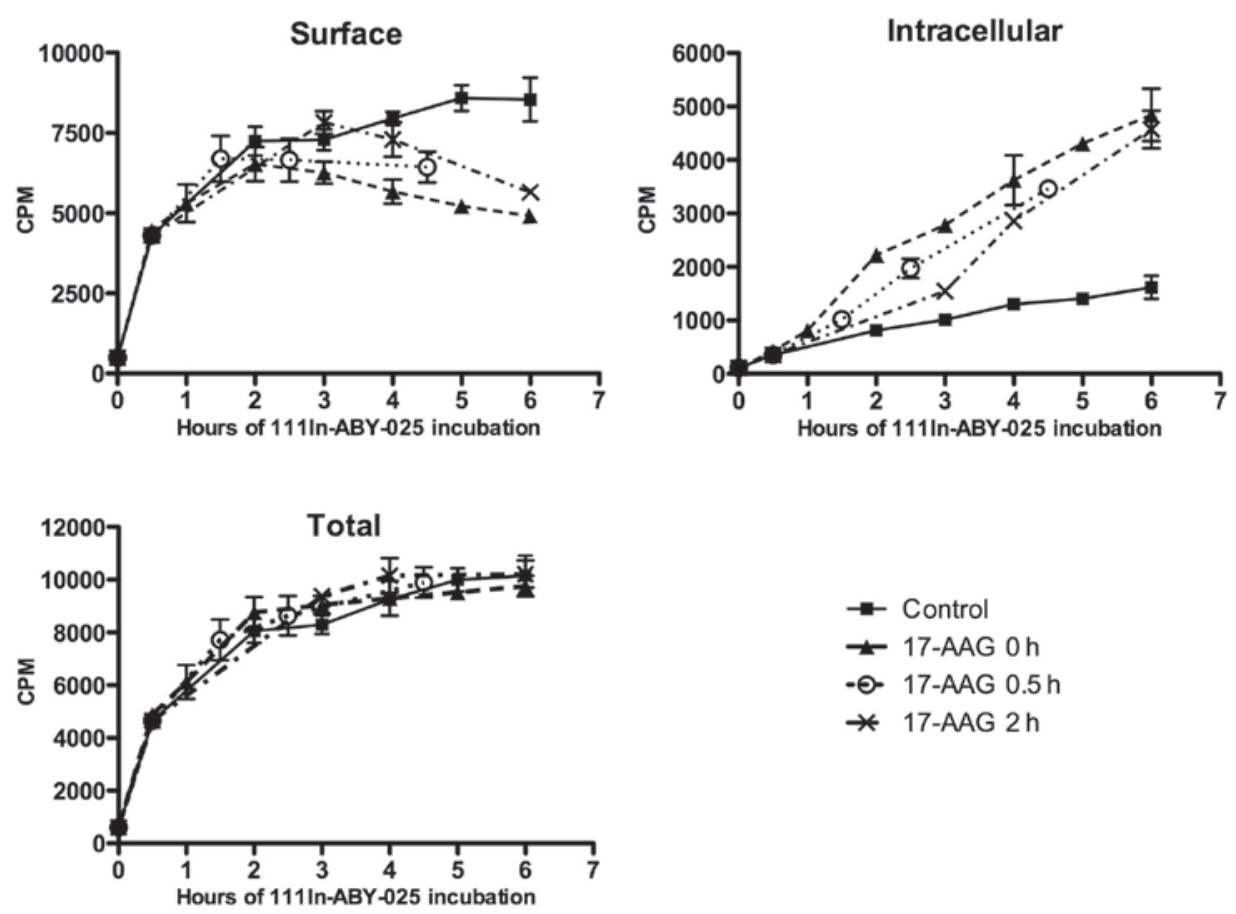

Figure 5. Cellular ${ }^{111}$ In-ABY-025 uptake and internalisation in untreated SKOV-3 cells (control, full line), cells treated with $100 \mathrm{nM} 17-\mathrm{AAG}$ after $0 \mathrm{~h}$ (dashed line with triangles), after $0.5 \mathrm{~h}$ (dotted line with circles) or after $2 \mathrm{~h}$ (dashed/dotted line with crosses) of incubation with ${ }^{111}$ In-ABY-025. Data are presented as the mean of three samples \pm standard deviation. cpm, counts per minute.

between radiometal- and halogen-labelled proteins has been demonstrated previously, and is probably a consequence of the lipophilicity, size and charge of the nuclide, linker and amino acid to which it is bound $(29,30)$.
The 17-AAG-induced internalisation of ABY-025 could be useful in several different applications. As long as the receptor binder is surface-bound, it is in equilibrium with the extracellular concentration of the binder. When extracel- 
lular concentration decreases, there is a risk of detachment of the binder, particularly if the internalisation rate is slow, as for HER2 (5). A triggered internalisation could increase the cellular retention of radionuclides provided that the nuclide is retained within the cell. It is well known, and was demonstrated in this study, that radiohalogens are excreted rapidly and are therefore not suitable for this approach (30). By contrast, radiometals are well-suited for this purpose due to their long intracellular retention. Furthermore, in a situation where metal radionuclides are used for therapeutic applications, efficacy could be improved by internalisation. The chance of a alpha or beta particle track from a radionuclide decay to ionise the DNA strand increases the closer to DNA the decay occurs due to pure geometry. A dose to the nucleus of a single cell will be higher if the radionuclide is positioned in the cytoplasm, compared with a dose from a radionuclide that is cell surface-bound (31).

The quality of the radiation, as well as the range of the particle emitted, will also be important factors determining the damage to the DNA of the cell (30). High LET from short-range particle tracks, such as $\alpha$-emitting nuclides, will produce more lethal double-strand breaks than from low LET $\beta$-emitting nuclides; however, only a few of the $\alpha$-emitting radionuclides fulfil the criteria for nuclear medicine applications, the most studied being ${ }^{211} \mathrm{At},{ }^{213} \mathrm{Bi},{ }^{225} \mathrm{Ac}$ and ${ }^{223} \mathrm{Ra}$ (32). With the exception of ${ }^{223} \mathrm{Ra}$, which is injected as the radium salt, all other proposed $\alpha$-emitting radionuclides require a tumour-specific carrier molecule. In the present study, ${ }^{211} \mathrm{At}$ was used for labelling of ABY-025. However, due to lack of residualising properties of At-211, a better choice in this case would likely be the $\alpha$-emitting metal radionuclide ${ }^{213} \mathrm{Bi}(32)$.

Other approaches, such as targeted cytotoxic agents, siRNAs or RNase, may also benefit from internalisation when aiming for HER2-expressing cells. Intracellular distribution of these agents is necessary to effectuate their therapeutic capacity. Although a method by which to release the agents from the lysosome to the cytoplasm, or even for further transport into the nucleus, must be determined, a common risk for all of the aforementioned approaches is the risk of triggering internalisation of HER2 before the receptor binder reaches the cells. As shown in the current study, when ${ }^{111} \mathrm{In}-\mathrm{ABY}-025$ was allowed to bind HER2 prior to 17-AAG treatment, the internalisation appeared to follow the same pattern as for simultaneous treatment. It therefore seems possible to avoid the risk of 17-AAG inducing HER2 internalisation and degradation before ABY-025 has bound to the receptor.

In conclusion, 17-AAG may be used to facilitate cellspecific intracellular localisation of a suitable cytotoxic or radioactive agent coupled to ABY-025 in HER2-overexpressing cells.17-AAG treatment of SKOV-3 and SKBR-3 cells to some extent shifts the localisation of 111In-ABY-025 from the cell surface to intracellular compartments in both cell lines. ABY-025 labelled with the high-LET alpha emitter 211At is also internalised to a higher degree, but due to its physiological properties this nuclide is excreted faster

\section{Acknowledgements}

The authors wish to thank Affibody AB for providing ABY-025; Associate Professor Kecke Elmroth and Helena
Kahu of Gothenburg University (Gothenburg, Sweden) for assisstance with experimental setups; and Professor Vladimir Tolmachev of Uppsala University for valuable discussions. This work was supported by the Swedish Cancer Society.

\section{References}

1. Slamon DJ, Godolphin W, Jones LA, Holt JA, Wong SG, Keith DE, Levin WJ, Stuart SG, Udove J and Ullrich A: Studies of the HER-2/neu proto-oncogene in human breast and ovarian cancer. Science 244: 707-712, 1989.

2. Carpenter G and Cohen S: 125I-labeled human epidermal growth factor. Binding, internalization and degradation in human fibroblasts. J Cell Biol 71: 159-171, 1976.

3. Stoscheck CM and Carpenter G: Down regulation of epidermal growth factor receptors: Direct demonstration of receptor degradation in human fibroblasts. J Cell Biol 98: 1048-1053, 1984.

4. Hendriks BS, Opresko LK, Wiley HS and Lauffenburger D: Coregulation of epidermal growth factor receptor/human epidermal growth factor receptor 2 (HER2) levels and locations: Quantitative analysis of HER2 overexpression effects. Cancer Res 63: 1130-1137, 2003.

5. Hommelgaard AM,Lerdrup M and van Deurs B: Association with membrane protrusions makes ErbB2 an internalization-resistant receptor. Mol Biol Cell 15: 1557-1567, 2004.

6. Worthylake R, Opresko LK and Wiley HS: ErbB-2 amplification inhibits down-regulation and induces constitutive activation of both ErbB-2 and epidermal growth factor receptors. J Biol Chem 274: 8865-8874, 1999.

7. Citri A, Skaria KB and Yarden Y: The deaf and the dumb: The biology of ErbB-2 and ErbB-3. Exp Cell Res 284: 54-65, 2003.

8. Tikhomirov $\mathrm{O}$ and Carpenter G: Geldanamycin induces ErbB-2 degradation by proteolytic fragmentation. J Biol Chem 275: 26625-26631, 2000.

9. Xu W, Mimnaugh E, Rosser MF, Nicchitta C, Marcu M, Yarden Y and Neckers L: Sensitivity of mature Erbb2 to geldanamycin is conferred by its kinase domain and is mediated by the chaperone protein Hsp90. J Biol Chem 276: 3702-3708, 2001.

10. Zhou P, Fernandes N, Dodge IL, Reddi AL, Rao N, Safran H, DiPetrillo TA, Wazer DE, Band V and Band H: ErbB2 degradation mediated by the co-chaperone protein CHIP. J Biol Chem 278: 13829-13837, 2003.

11. Raja SM, Clubb RJ, Bhattacharyya M, Dimri M, Cheng H, Pan W, Ortega-Cava C, Lakku-Reddi A, Naramura M, Band V and Band $\mathrm{H}$ : A combination of Trastuzumab and 17-AAG induces enhanced ubiquitinylation and lysosomal pathway-dependent ErbB2 degradation and cytotoxicity in ErbB2-overexpressing breast cancer cells. Cancer Biol Ther 7: 1630-1640, 2008.

12. Lerdrup M, Hommelgaard AM, Grandal M and van Deurs B: Geldanamycin stimulates internalization of ErbB2 in a proteasome-dependent way. J Cell Sci 119: 85-95, 2006.

13. Austin CD, De Mazière AM, Pisacane PI, van Dijk SM, Eigenbrot C, Sliwkowski MX, Klumperman J and Scheller RH: Endocytosis and sorting of ErbB2 and the site of action of cancer therapeutics trastuzumab and geldanamycin. Mol Biol Cell 15: 5268-5282, 2004.

14. Powers MV and Workman P: Targeting of multiple signalling pathways by heat shock protein 90 molecular chaperone inhibitors. Endocr Relat Cancer 13 (Supply 1): S125-S135, 2006.

15. Fukuyo Y, Hunt CR and Horikoshi N: Geldanamycin and its anti-cancer activities. Cancer Lett 290: 24-35, 2010.

16. Sain N, Krishnan B, Ormerod MG, De Rienzo A, Liu WM, Kaye SB, Workman P and Jackman AL: Potentiation of paclitaxel activity by the HSP90 inhibitor 17-allylamino-17-demethoxygeldanamycin in human ovarian carcinoma cell lines with high levels of activated AKT. Mol Cancer Ther 5: 1197-1208, 2006.

17. Banerji U, Sain N, Sharp SY, Valenti M, Asad Y, Ruddle R, Raynaud F, Walton M, Eccles SA, Judson I, et al: An in vitro and in vivo study of the combination of the heat shock protein inhibitor 17-allylamino-17-demethoxygeldanamycin and carboplatin in human ovarian cancer models. Cancer Chemother Pharmacol 62: 769-778, 2008.

18. Hu L, Hofmann J, Lu Y, Mills GB and Jaffe RB: Inhibition of phosphatidylinositol 3'-kinase increases efficacy of paclitaxel in in vitro and in vivo ovarian cancer models. Cancer Res 62: 1087-1092, 2002. 
19. Wu X, Wanders A, Wardega P, Tinge B, Gedda L, Bergstrom S, Sooman L, Gullbo J, Bergqvist M, Hesselius P, et al: Hsp90 is expressed and represents a therapeutic target in human oesophageal cancer using the inhibitor 17-allylamino-17-demethoxygeldanamycin. Br J Cancer 100: 334-343, 2009.

20. Nygren PA: Alternative binding proteins: Affibody binding proteins developed from a small three-helix bundle scaffold. FEBS J 275: 2668-2676, 2008

21. Friedman M and Ståhl S: Engineered affinity proteins for tumour-targeting applications. Biotechnol Appl Biochem 53. $1-29,2009$.

22. Baum RP, Prasad V, Müller D, Schuchardt C, Orlova A, Wennborg A, Tolmachev V and Feldwisch J: Molecular imaging of HER2expressing malignant tumors in breast cancer patients using synthetic 111In- or 68Ga-labeled affibody molecules. J Nucl Med 51: 892-897, 2010

23. Ahlgren S, Orlova A, Wallberg H, Hansson M, Sandström M, Lewsley R, Wennborg A, Abrahmsén L, Tolmachev V and Feldwisch J: Targeting of HER2-expressing tumors using 111In-ABY-025, a second-generation affibody molecule with a fundamentally reengineered scaffold. J Nucl Med 51: 1131-1138, 2010.

24. Lindegren S, Frost S, Bäck T, Haglund E, Elgqvist J and Jensen H: Direct procedure for the production of 211 At-labeled antibodies with an epsilon-lysyl-3 (trimethylstannyl)benzamide immunoconjugate. J Nucl Med 49: 1537-1545, 2008.

25. Wallberg $\mathrm{H}$ and Orlova A: Slow internalization of anti-HER2 synthetic affibody monomer 111In-DOTA-ZHER2:342-pep2: Implications for development of labeled tracers. Cancer Biother Radiopharm 23: 435-442, 2008.
26. Gartner EM, Silverman P, Simon M, Flaherty L, Abrams J, Ivy P, Lorusso PM: A phase II study of 17-allylamino-17-demethoxygeldanamycin in metastatic or locally advanced, unresectable breast cancer. Breast Cancer Res Treat 131(3):933-7, 2012.

27. Pedersen KS, Kim GP, Foster NR, Wang-Gillam A, Erlichman C, McWilliams RR: Phase II trial of gemcitabine and tanespimycin (17AAG) in metastatic pancreatic cancer: a Mayo Clinic Phase II Consortium study. Invest New Drugs. 33(4):963-8, 2015.

28. Oki Y, Copeland A, Romaguera J, Fayad L, Fanale M, Faria Sde C, Medeiros LJ, Ivy P, Younes A: Clinical experience with the heat shock protein-90 inhibitor, tanespimycin, in patients with relapsed lymphoma. Leuk Lymphoma 53(5):990-2, 2012.

29. Shih LB, Thorpe SR, Griffiths GL, Diril H, Ong GL, Hansen HJ, Goldenberg DM and Mattes MJ: The processing and fate of antibodies and their radiolabels bound to the surface of tumor cells in vitro: A comparison of nine radiolabels. J Nucl Med 35: 899-908, 1994

30. Tolmachev V: Chapter 8: Choice of Radionuclides and Radiolabelling Techniques. In: Targeted Radionuclide Tumor Therapy - Biological Aspects. Stigbrand T, Carlsson J and Adams GP (eds). Springer, pp145-174, 2008.

31. Hartman T, Lundqvist H, Westlin JE and Carlsson J: Radiation doses to the cell nucleus in single cells and cells in micrometastases in targeted therapy with (131)I labeled ligands or antibodies. Int J Radiat Oncol Biol Phys 46: 1025-1036, 2000.

32. Wilbur S: Chemical and Radiochemical Considerations in Radiolabeling with-Emitting Radionuclides. Current Pharmaceuticals 4: 214-247, 2011. 